\title{
Neoadjuvant immunotherapy plus chemotherapy achieved pathologic complete response in stage IIIB lung adenocarcinoma harbored EGFR G779F: a case report
}

\author{
Yadong Wang ${ }^{1,2}$, Xiaoying Yang ${ }^{1,2}$, Xu Tian ${ }^{3}$, Ziqi Jia ${ }^{1,2}$, Zhongxing Bing ${ }^{1}$, Lei Cao ${ }^{1}$, Chao Gao ${ }^{1}$, \\ Zhili Cao ${ }^{1}$, Shanqing Li $^{1}$, Naixin Liang ${ }^{1}$ \\ ${ }^{1}$ Department of Thoracic Surgery, Peking Union Medical College Hospital, Peking Union Medical College and Chinese Academy of Medical \\ Sciences, Beijing, China; ${ }^{2}$ Peking Union Medical College, Chinese Academy of Medical Sciences, Beijing, China; ${ }^{3}$ Department of Otolaryngology, \\ Peking Union Medical College Hospital, Peking Union Medical College and Chinese Academy of Medical Sciences, Beijing, China \\ Correspondence to: Naixin Liang. Peking Union Medical College Hospital, No.1 Shuaifuyuan, Dongcheng District, Beijing 100730, China. \\ Email: pumchnelson@163.com.
}

\begin{abstract}
The majority of patients with lung cancer are in the late stage (stages IIIB or IV) when diagnosed. However, clinical features, treatment and prognosis of some patients in stage IIIB are somewhat different from those in stage IV. Several clinical trials of neoadjuvant immunotherapy are changing the treatment strategy for patients with stage IB-IIIA non-small cell lung cancer (NSCLC). It remains unclear whether patients with stage IIIB NSCLC could benefit from neoadjuvant immunotherapy because they are often excluded from clinical trials. The IMpower150 trial showed promising results in the clinics of atezolizumab plus bevacizumab plus chemotherapy in patients with epidermal growth factor receptor (EGFR) mutation positive NSCLC. However, reports of this treatment strategy on EGFR exon 20 mutations are still lacking. Osimertinib is effective for T790M mutation but results of targeted therapy in other EGFR exon 20 mutations are not favorable and there is currently no effective long-term therapy. Patients harboring EGFR exon 20 G779F mutation have not been reported to achieve a complete response (CR). Here, we report the case of a 55-year-old man who was diagnosed as stage IIIB (cT1bN3M0) pulmonary adenocarcinoma by supraclavicular lymph node biopsy. He was administered chemotherapy plus durvalumab before surgery. The disease was considered as a partial response (PR), and the postoperative pathology revealed that a pathologic CR had been achieved. At the time of writing, no signs of recurrence had been observed in the preceding 15 months. Our case provides a new treatment option for such patients.
\end{abstract}

Keywords: Lung cancer; neoadjuvant immunotherapy; N3 lymph node metastasis; uncommon epidermal growth factor receptor mutation (uncommon EGFR mutation); case report

Submitted Apr 29, 2020. Accepted for publication Sep 25, 2020.

doi: 10.21037/apm-20-1692

View this article at: http://dx.doi.org/10.21037/apm-20-1692

\section{Introduction}

One-third of non-small cell lung cancer (NSCLC) patients are staged as locally advanced at the time of initial diagnosis (1). For patients with unresectable stage III NSCLC, concurrent chemoradiation is the current standard of care; for patients who respond to treatment, this is followed by consolidation immunotherapy with durvalumab (2). However, because of the clinical heterogeneity of stage III disease, the definitions of unresectability vary among surgeons. With the advent of effective systemic immunotherapy, surgical intervention followed by immunotherapy for patients with locally advanced disease is emerging as a new therapeutic option.

Previously, the efficacy and feasibility of neoadjuvant immunotherapy before tumor resection in patients with early-stage resectable NSCLC has been demonstrated (3). 
The NADIM study, a phase II trial involving 46 patients with stage IIIA (N2) NSCLC, examined neoadjuvant chemotherapy plus nivolumab followed by adjuvant nivolumab for 1 year, revealing an unusually high objective response rate (4). Forty-one surgeries had been performed, with $34(83 \%)$ patients showing major pathologic response (MPR) and 24 (71\%) showing pathological complete response (pCR). These results are a reminder that some patients with stage IIIB (N3) disease might also benefit from neoadjuvant immunotherapy, which holds promise for improving the long-term prognosis.

Although the NADIM study reported encouraging results following neoadjuvant immunotherapy, the study excluded patients with epidermal growth factor receptor (EGFR) mutations. The most common EGFR mutations are exon 19 deletions and exon 21 Leu858Arg mutation (L858R). These mutations are usually termed classical mutations and account for more than $90 \%$ of all EGFR mutations. In addition to classical mutations, other rare EGFR mutations within exons 18 to 21 are known as uncommon EGFR mutations. At present, EGFR tyrosine kinase inhibitors (TKIs) are the standard first-line treatment for patients harboring classic EGFR mutations. However, the efficacy of treatment targeting some uncommon EGFR mutations is far from satisfactory. More effective and specific therapies for these patients urgently need to be developed.

Neoadjuvant immunotherapy plus chemotherapy followed by surgery may offer an advantageous or alternative method for treating stage IIIB NSCLC with uncommon EGFR mutations. Here, we report the case of a patient with initially inoperable IIIB (cT1bN3M0) NSCLC with EGFR G779F mutation. After receiving a combination of platinum-based chemotherapy plus durvalumab, the patient became operable and underwent video-assisted thoracoscopic surgery (VATS) right upper lung lobectomy plus radical mediastinal lymph node dissection. He went on to achieve at least 15 months' disease-free survival (DFS). We present the following case in accordance with the CARE reporting checklist (available at http://dx.doi. org/10.21037/apm-20-1692).

\section{Case presentation}

In May 2019, a 55-year-old male with a history of heavy smoking (smoking index 600) was admitted to our hospital with a palpable left supraclavicular lymph nodule. The patient did not report experiencing any chest pain, hemoptysis, hoarseness, night sweats, or significant loss of body weight. The patient's Eastern Cooperative Oncology Group (ECOG) performance status was 0. He had no significant medical or family history. Laboratory tests showed the patient's serum tumor marker levels to be: carcinoembryonic antigen (CEA) $251.8 \mathrm{ng} / \mathrm{mL}$, cytokeratin-19 fragment (CYFRA 21-1) $7.3 \mathrm{ng} / \mathrm{mL}$, and carbohydrate antigen 125 (CA125) $333.3 \mathrm{U} / \mathrm{mL}$. Positron-emission tomography/computed tomography (PET/CT) revealed a $19 \mathrm{~mm}$ right upper lobe nodule with a standardized uptake value (SUV) max of 7.8. High metabolic right hilar and mediastinal as well as left supraclavicular lymph nodes were also observed (Figure 1). There was no evidence of distant metastasis.

An excisional biopsy of the left supraclavicular lymph node showed metastatic lung adenocarcinoma (Figure 1). The patient was clinically diagnosed as inoperable stage IIIB (cT1bN3M0) lung adenocarcinoma, according to the 8th American Joint Committee on Cancer (AJCC) classification. A median survival of 10 months was predicted (5). Molecular pathologic analysis revealed EGFR exon 20 G779F mutation at $69.2 \%$ allelic frequency and EGFR amplification without additional targetable mutations or immune-related genes of interest. The overall tumor mutation burden (TMB) was 7.68 Muts/Mb. Moreover, the clone Dako 22C3 pharmDx antibody was used, and the expression of programmed cell death-ligand 1 (PD-L1) was found to be $20 \%$ (Figure 1).

After extensively discussing standard treatment options and the potential risks of pursuing a non-standard treatment strategy with the patient and his family, a combination of immunotherapy and chemotherapy was adopted. The patient was started on gemcitabine $\left(1,250 \mathrm{mg} / \mathrm{m}^{2}\right)$ and cisplatin $\left(80 \mathrm{mg} / \mathrm{m}^{2}\right)$ combined with durvalumab $(10 \mathrm{mg} / \mathrm{kg})$, administered every 3 weeks for 2 cycles between July and August, 2019. The adverse events were tolerable and well controlled. No grade 4 toxicities were recorded during the neoadjuvant therapy. Post-treatment radiologic evaluation by enhanced CT scan showed that compared with the disease status in May 2019, the right upper lobe nodule had decreased in size (Figure 2). The disease was considered as partial response (PR), according to the Response Evaluation Criteria in Solid Tumors (RECIST) version 1.1 (6).

Following multidisciplinary evaluation and discussion, 4 weeks after completing neoadjuvant therapy, the patient underwent VATS right upper lung lobectomy plus radical mediastinal lymph node dissection. The postoperative pathologic results revealed no residual viable tumor cells 

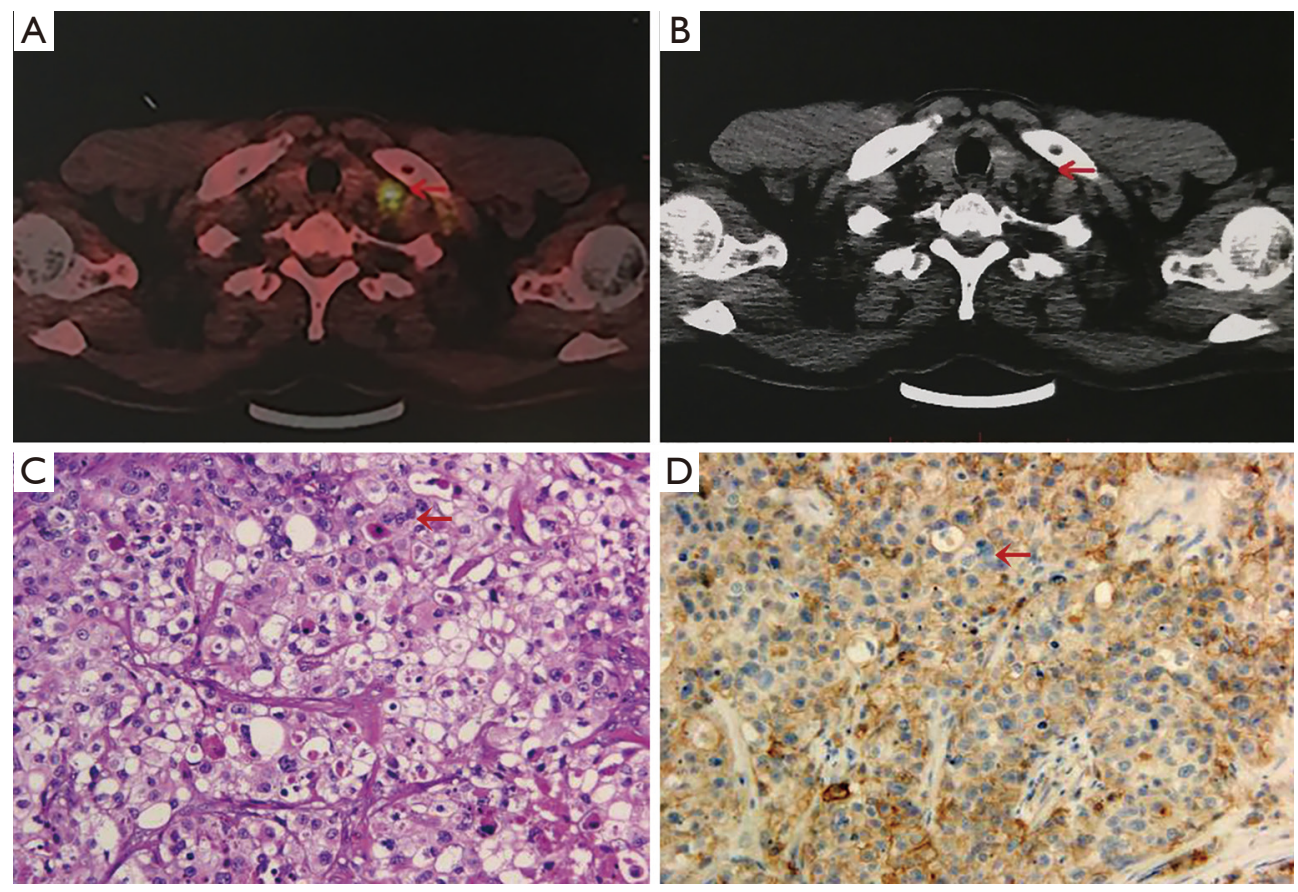

Figure 1 Radiological and pathological findings of the left supraclavicular nodule. (A) PET/CT and (B) CT scan image of the left supraclavicular lymph nodes (red arrow); (C) HE-stained image of the excised left supraclavicular lymph nodes. (Original magnification, $\times 200$ ); (D) PD-L1 assay of the excised left supraclavicular lymph nodes by immunohistochemical staining. (Original magnification, $\times 200)$. PET/CT, positron-emission tomography/computed tomography; HE, hematoxylin and eosin; PD-L1, programmed cell death-ligand 1.
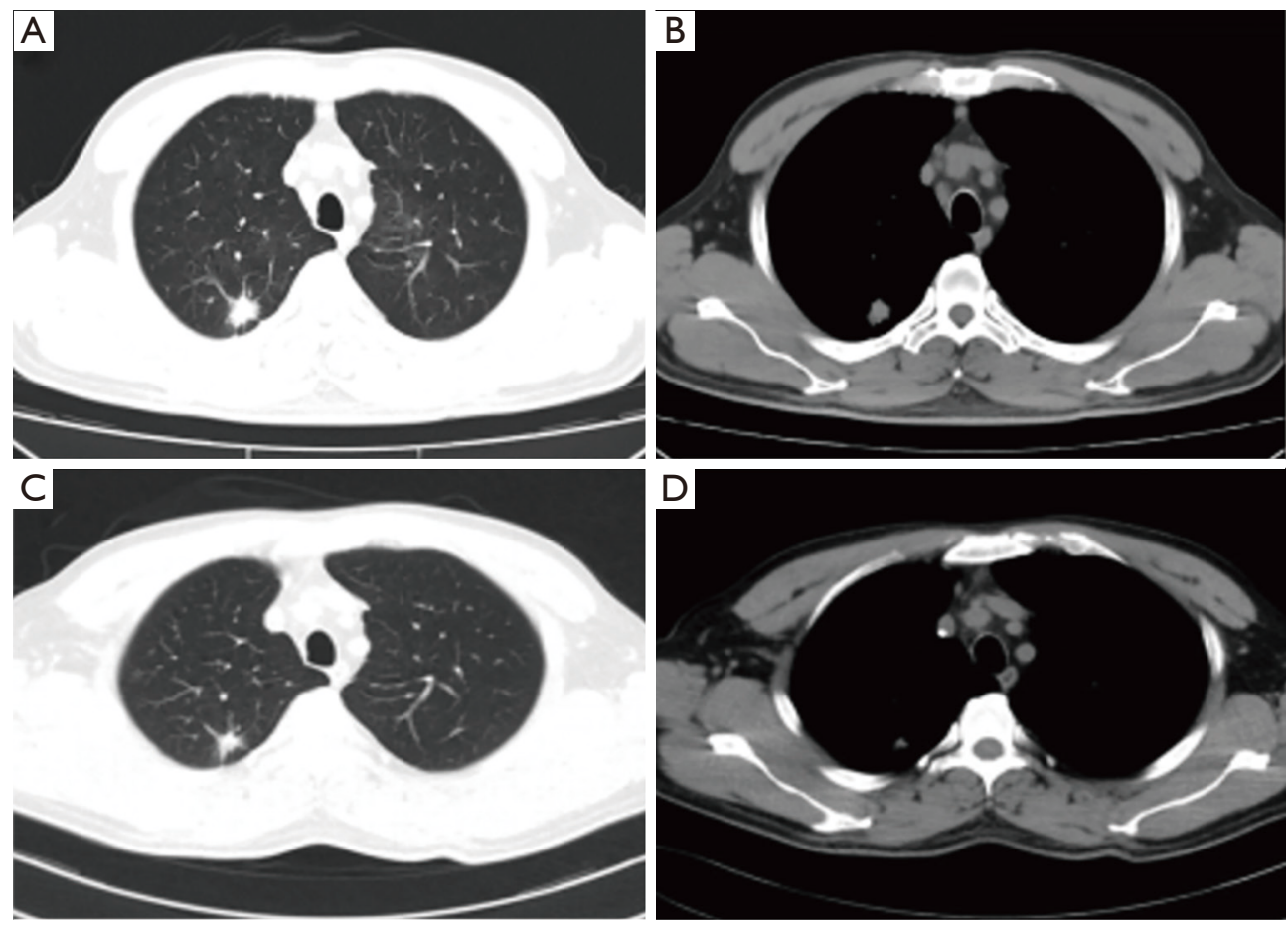

Figure 2 Radiological evaluation of the treatment efficacy with CT at distinct time points. (A) and (B) show chest CT images before neoadjuvant therapy; and (C) and (D) show chest CT images after two cycles of treatment. CT, computed tomography. 


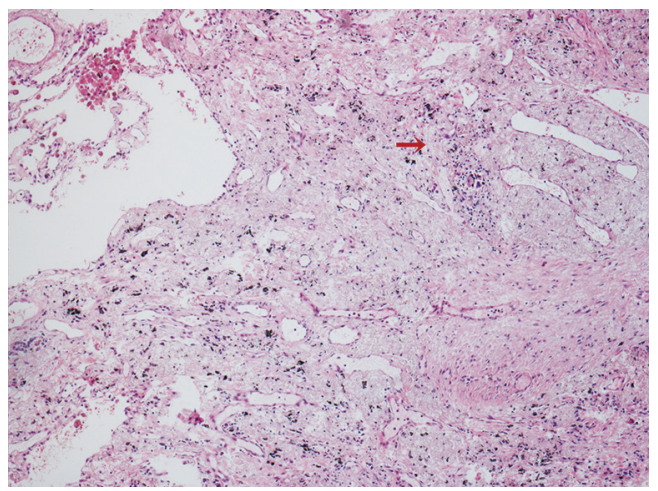

Figure 3 Postoperative pathological HE-stained picture of the primary lesion of the right upper lung lobe. (original magnification $\times 60)$. HE, hematoxylin and eosin.

in the primary lung cancer lesion, confirming that the patient had achieved pCR after neoadjuvant treatment (Figure 3) (7). Surgical margins and lymph nodes at levels $2 \mathrm{R}, 4 \mathrm{R}, 7,10,11$, and 12 as well as the right upper paratracheal lymph node tested negative for carcinoma. The patient was discharged on postoperative day 3 after recovering with no perioperative complications.

The patient was administered adjuvant gemcitabine and cisplatin combined with durvalumab for 2 cycles 2 months after the operation. He underwent clinical and radiologic assessments every 3 months, and a recent CT scan examination found no evidence of recurrence or metastasis. The serum biomarkers of CEA, CA125, and CYFRA21-1 decreased to normal values. Peripheral blood samples $(2 \mathrm{~mL})$ were collected from the patient before the initiation of neoadjuvant immunotherapy and at a recent followup. The numbers of circulating tumor cells (CTCs) and PD-L1-positive CTCs were analyzed, showing a decrease from 10 to 3 in the total number of CTCs and a decrease from 10 to 1 in the number of PD-L1-positive CTCs. The patient was also checked for NSCLC by tracking the EGFR G779F mutation in circulating tumor DNA (ctDNA). Plasma analysis at baseline showed EGFR G779F ctDNA, which was undetectable at follow-up. At the time of writing, no signs of recurrence had been observed in the 15 months prior (Figure 4). However, long-term follow-up is still needed to confirm the effectiveness of the treatment strategy.

All procedures performed in studies involving human participants were in accordance with the ethical standards of the institutional and/or national research committee(s) and with the Helsinki Declaration (as revised in 2013). All treatments were performed with the consent of the patient. Written informed consent was obtained from the patient for publication of this study and any accompanying images.

\section{Discussion}

Stage III NSCLC patients are an extremely heterogeneous group, and a single therapeutic concept cannot meet the needs of all subsets of patients. In a proportion of cases, stage IIIB NSCLC has been observed to metastasize to distant lymph nodes (N3) while the primary tumor is still small. Stage IIIB NSCLC with lymph node metastasis is highly aggressive and may involve unique biological pathways. Based on current clinical guidelines, patients with the disease are not surgically resectable with curative intent. The average 5-year survival rate of patients with N3 lymph node metastasis has been reported as $9 \%$, according to data released by the International Association for the Study of Lung Cancer (IASLC) in 2015 (5). For these patients, effective therapies are urgently needed.

Numerous attempts to improve the therapeutic outcomes have been made. nevertheless, the survival of patients is still unsatisfactory. A previous study of patients with locally advanced NSCLC found that preoperative platinumbased chemotherapy only provided a $5 \%$ increase in the 5 -year survival rate compared with surgery alone. With the incidence of toxicities of grade 3 or higher exceeding $60 \%$, its use is limited (8). No significant difference in survival benefit was found between patients who received neoadjuvant chemotherapy and those who received adjuvant chemotherapy. Neoadjuvant immunotherapy with antiPD-1/PD-L1 targeting antibodies has recently produced highly encouraging results and holds promising therapeutic efficacy in NSCLC $(3,4,9,10)$. In particular, unexpectedly high pathologic response rates were observed in patients with stage IIIA (N2) disease in the NADIM study, which prompts the question of whether patients with stage IIIB (N3) disease could also benefit from such management.

Our patient was treated with neoadjuvant immunotherapy using platinum-based chemotherapy plus durvalumab for two cycles before surgery. The aims of neoadjuvant immunotherapy are to eliminate distant occult metastasis and to downstage the primary tumor and lymph node metastasis. PCR was observed after pathological evaluation of the surgical specimen. In a study of neoadjuvant chemotherapy for nonsquamous NSCLC, 


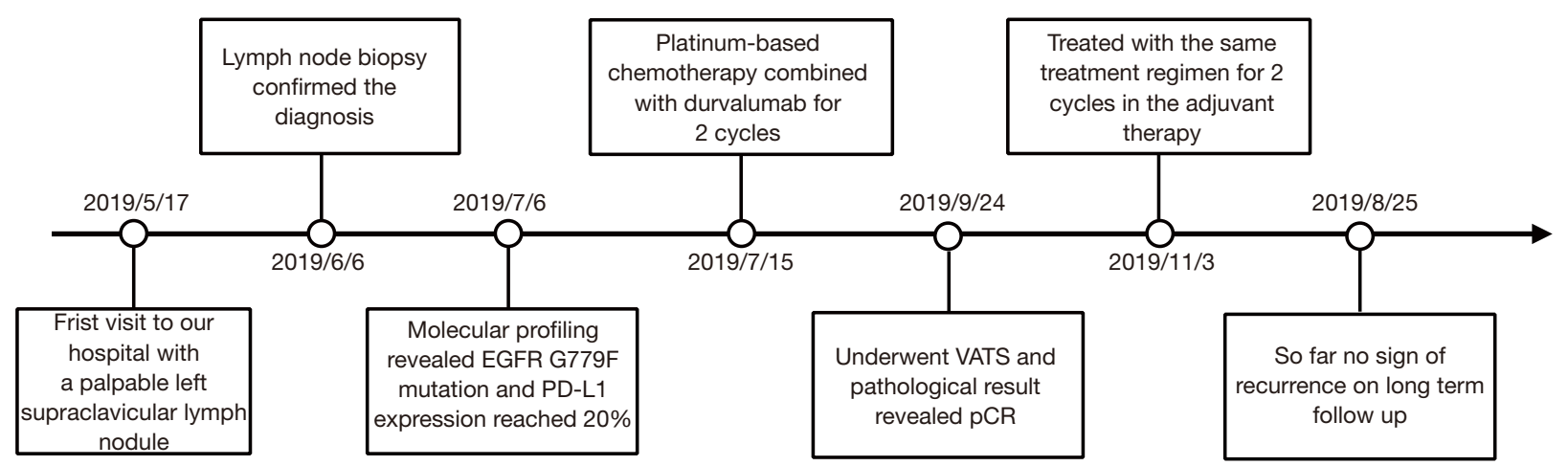

Figure 4 Timeline of diagnosis, treatment and prognosis in this case. EGFR, epidermal growth factor receptor; PD-L1, programmed cell death-ligand 1; VATS, video-assisted thoracoscopic surgery; pCR, pathological complete response.

such responses were positively correlated with long-term survival benefit (11). Another retrospective analysis also showed that the occurrence of pCR was associated with improved recurrence-free and overall survival (12). This case demonstrated that chemotherapy plus immunotherapy could downstage some patients with unresectable N3 NSCLC to operable status. Therefore, handpicking N3 patients who might benefit from this new therapeutic strategy should be encouraged. Further prospective multicenter clinical trials will be needed to validate the treatment strategy.

Despite the earlier encouraging results, many questions have yet to be resolved regarding patients with stage IIIB NSCLC. One of the most critical issues is determining those patients who could benefit most from neoadjuvant therapies. The 8th edition of TNM staging for lung cancer updated the $\mathrm{T}$ descriptors for patients with $\mathrm{N} 3$ disease, separately classifying those with T1-2N3 (stage IIIB) and those with T3-4N3 (stage IIIC) disease (1). Statistically significance differences between stage IIIB and stage IIIC patients, as reported by the IASLC, were confirmed in a more extensive population-based analysis using data from the National Cancer Institute (13). Patients with stage T1N3 may potentially benefit from neoadjuvant immunotherapy with consideration for the biological characteristics of the tumor.

Along with clinical phenotypes, molecular characteristics should also be comprehensively considered. Genetic testing and targeted therapy based on EGFR and anaplastic lymphoma kinase $(A L K)$ gene mutation status have been recommended in clinical practice guidelines. PD-L1 and TMB have been found to be associated with the efficacy of immunotherapy. These molecular characteristics are important for clinical decision making and for selecting treatment of individual patients. At present, immunohistochemical staining of tumor tissue with PD-L1 antibodies can be used to predict the therapeutic response of immunotherapy to some extent. Additionally, TMB is emerging as a practical biomarker for predicting the efficacy of immune checkpoint inhibitors. As these two candidate biomarkers have mainly been examined in patients with advanced lung cancer, several clinical trials have begun to explore predictive biomarkers for neoadjuvant immunotherapy. The CheckMate-159 study first explored the efficacy and safety of neoadjuvant immunotherapy for stage I-IIIA NSCLC, finding that MPR was significantly correlated with TMB before treatment but not with PD-L1 expression (3). In contrast, mid-term analysis of the clinical trial LCMC3 reported that MPR was independent of PD-L1 expression and TMB (10). However, the subgroup analysis of the NEOSTAR study found that the PD-L1 expression level was related to MPR (9). Taken together, the potential of PD-L1 expression and TMB as predictors for screening patients with lung cancer for neoadjuvant immunotherapies calls for further exploration. A recent clinical study, ChiCTR-OIC-17013726, for the first time introduced baseline and preoperative PET/CT scans to evaluate tumor response after sintilimab treatment in the neoadjuvant setting (14). Decreases in $\mathrm{SUV}_{\text {max }}$ were correlated with postoperative MPR status, which may aid in the assessment of tumor pathologic response (15). Furthermore, PD-L1 expression on stromal cells instead of tumor cells was related to pathologic regression. All of the above evidence comes from several small-scale clinical trials. Thus, large-scale phase III studies are required in the future to help us better understand the response to neoadjuvant immunotherapies and to establish potential biomarkers for identifying patients who would benefit from such treatments. 
The EGFR alteration of our patient is another point deserving of attention. Immunotherapy is widely understood to be less effective in patients with EGFR mutations than in patients with wild-type EGFR $(16,17)$. Therefore, the NCCN guidelines recommend targeted drug treatment for patients with EGFR mutations who cannot undergo surgery. However, a small amount of research has shown that some EGFR mutation-positive patients might also benefit from treatment with PD-1/PD-L1 inhibitors. The phase III IMpower150 study showed encouraging clinical activity of atezolizumab plus bevacizumab plus carboplatin plus paclitaxel (ABCP) in patients with EGFR-positive NSCLC (18). Another small-scale pilot study showed that uncommon EGFR mutations were the independent predictive factor for the efficacy of nivolumab (19). Our patient carried the EGFR exon 20 G779F mutation, which is categorized as an uncommon mutation which might not benefit from EGFR-targeted therapies. Furthermore, the patient's PD-L1 expression reached 20\%, which suggested that he could potentially benefit from immunotherapy. Thus, after a multidisciplinary discussion, a combination of platinum-based chemotherapy plus durvalumab was chosen. This new potential strategy achieved stable effects and satisfactory results. Neoadjuvant immunotherapy plus chemotherapy followed by surgery may offer an alternative treatment strategy for patients with stage IIIB NSCLC who have uncommon EGFR mutations and positive PDL1 expression. However, our findings need to be verified in further studies.

In conclusion, our case shows the successful treatment of a patient with initially inoperable IIIB (cT1bN3M0) NSCLC with EGFR G779F mutation, who became operable after the use of platinum-based chemotherapy combined with durvalumab. Future strategies to optimize treatment for handpicked patients with NSCLC and $\mathrm{N} 3$ involvement should focus on both molecular and clinical phenotypes. Close multidisciplinary collaboration plays a vital role in identifying proper and more specific management approaches to improve the outcomes of patients with this aggressive disease.

\section{Acknowledgments}

Funding: This work was supported by the National Key Research and Development Program of China (No. 2016YFC0901500), Beijing Health Promotion Association (BJHPA-FW-XHKT-2020040400344), Beijing Natural
Science Foundation (7182132), Major projects of the Beijing Municipal Science and Technology Commission (Z171100002017013), and Beijing Dongcheng District Science and Technology Commission Excellent Talents Project (2018-DCQYXRCXM-012).

\section{Footnote}

Reporting Checklist: The authors have completed the CARE reporting checklist. Available at http://dx.doi.org/10.21037/ apm-20-1692

Conflicts of Interest: All authors have completed the ICMJE uniform disclosure form (available at http://dx.doi. org/10.21037/apm-20-1692). The authors have no conflicts of interest to declare.

Etbical Statement: The authors are accountable for all aspects of the work in ensuring that questions related to the accuracy or integrity of any part of the work are appropriately investigated and resolved. All procedures performed in studies involving human participants were in accordance with the ethical standards of the institutional and/or national research committee(s) and with the Helsinki Declaration (as revised in 2013). All treatments were performed with the consent of the patient. Written informed consent was obtained from the patient for publication of this study and any accompanying images.

Open Access Statement: This is an Open Access article distributed in accordance with the Creative Commons Attribution-NonCommercial-NoDerivs 4.0 International License (CC BY-NC-ND 4.0), which permits the noncommercial replication and distribution of the article with the strict proviso that no changes or edits are made and the original work is properly cited (including links to both the formal publication through the relevant DOI and the license). See: https://creativecommons.org/licenses/by-nc-nd/4.0/.

\section{References}

1. Goldstraw P, Chansky K, Crowley J, et al. The IASLC Lung Cancer Staging Project: Proposals for Revision of the TNM Stage Groupings in the Forthcoming (Eighth) Edition of the TNM Classification for Lung Cancer. J Thorac Oncol 2016;11:39-51.

2. Antonia SJ, Villegas A, Daniel D, et al. Overall Survival 
with Durvalumab after Chemoradiotherapy in Stage III NSCLC. N Engl J Med 2018;379:2342-50.

3. Forde PM, Chaft JE, Smith KN, et al. Neoadjuvant PD-1 Blockade in Resectable Lung Cancer. N Engl J Med 2018;378:1976-86.

4. Provencio M, Nadal E, Insa A, et al. Neoadjuvant chemo-immunotherapy for the treatment of stage IIIA resectable non-small-cell lung cancer (NSCLC): A phase II multicenter exploratory study-Final data of patients who underwent surgical assessment. J Clin Oncol 2019;37:abstr 8509.

5. Asamura H, Chansky K, Crowley J, et al. The International Association for the Study of Lung Cancer Lung Cancer Staging Project: Proposals for the Revision of the N Descriptors in the Forthcoming 8th Edition of the TNM Classification for Lung Cancer. J Thorac Oncol 2015;10:1675-84.

6. Eisenhauer EA, Therasse P, Bogaerts J, et al. New response evaluation criteria in solid tumours: revised RECIST guideline (version 1.1). Eur J Cancer 2009;45:228-47.

7. Hellmann MD, Chaft JE, William WN, et al. Pathological response after neoadjuvant chemotherapy in resectable non-small-cell lung cancers: proposal for the use of major pathological response as a surrogate endpoint. Lancet Oncol 2014;15:e42-e50.

8. Preoperative chemotherapy for non-small-cell lung cancer: a systematic review and meta-analysis of individual participant data. Lancet 2014;383:1561-71.

9. Cascone T, William WN, Weissferdt A, et al. Neoadjuvant nivolumab $(\mathrm{N})$ or nivolumab plus ipilimumab (NI) for resectable non-small cell lung cancer (NSCLC): Clinical and correlative results from the NEOSTAR study. J Clin Oncol 2019;37:abstr 8504.

10. Kwiatkowski DJ, Rusch VW, Chaft JE, et al. Neoadjuvant atezolizumab in resectable non-small cell lung cancer (NSCLC): Interim analysis and biomarker data from a multicenter study (LCMC3). J Clin Oncol 2019;37:abstr 8503.

11. Chaft JE, Rusch V, Ginsberg MS, et al. Phase II trial of

Cite this article as: Wang $\mathrm{Y}$, Yang $\mathrm{X}$, Tian $\mathrm{X}$, Jia Z, Bing Z, Cao L, Gao C, Cao Z, Li S, Liang N. Neoadjuvant immunotherapy plus chemotherapy achieved pathologic complete response in stage IIIB lung adenocarcinoma harbored EGFR G779F: a case report. Ann Palliat Med 2020;9(6):4339-4345. doi: 10.21037/apm-20-1692 neoadjuvant bevacizumab plus chemotherapy and adjuvant bevacizumab in patients with resectable nonsquamous nonsmall-cell lung cancers. J Thorac Oncol 2013;8:1084-90.

12. Pataer A, Kalhor N, Correa AM, et al. Histopathologic response criteria predict survival of patients with resected lung cancer after neoadjuvant chemotherapy. J Thorac Oncol 2012;7:825-32.

13. Shin JY, Yoon JK, Marwaha G. External Validation of the New International Association for the Study of Lung Cancer Tumor, Node, and Metastasis 8th Edition Staging System and Updated T Descriptors in Determining Prognosis for Patients With Non-Small Cell Lung Cancer Patients With N3 Disease. Clin Lung Cancer 2017;18:e481-9.

14. Gao S, Li N, Gao S, et al. Neoadjuvant PD-1 inhibitor (Sintilimab) in NSCLC. J Thorac Oncol 2020;15:816-26.

15. Tao X, Li N, Wu N, et al. The efficiency of F-FDG PET-CT for predicting the major pathologic response to the neoadjuvant PD-1 blockade in resectable nonsmall cell lung cancer. Eur J Nucl Med Mol Imaging 2020;47:1209-19.

16. Lee CK, Man J, Lord S, et al. Checkpoint Inhibitors in Metastatic EGFR-Mutated Non-Small Cell Lung Cancer-A Meta-Analysis. J Thorac Oncol 2017;12:403-7.

17. Lee CK, Man J, Lord S, et al. Clinical and Molecular Characteristics Associated With Survival Among Patients Treated With Checkpoint Inhibitors for Advanced NonSmall Cell Lung Carcinoma: A Systematic Review and Meta-analysis. JAMA Oncol 2018;4:210-6.

18. Reck M, Mok TSK, Nishio M, et al. Atezolizumab plus bevacizumab and chemotherapy in non-small-cell lung cancer (IMpower150): key subgroup analyses of patients with EGFR mutations or baseline liver metastases in a randomised, open-label phase 3 trial. Lancet Respir Med 2019;7:387-401.

19. Yoshida H, Kim YH, Ozasa H, et al. Nivolumab in nonsmall-cell lung cancer with EGFR mutation. Ann Oncol 2018;29:777-8. 